\title{
FROM UNCERTAINTY PRINCIPLES TO WEGNER ESTIMATES
}

\author{
PETER STOLLMANN ${ }^{\dagger}$
}

\begin{abstract}
We give a shortcut from simple uncertainty principles to Wegner estimates with correct volume term.
\end{abstract}

Dedicated to the memory of Pierre Duclos.

\section{INTRODUCTION}

The path described in the title has been laid by Combes, Hislop and Klopp, [5] to prove Wegner estimates with the correct volume factor for Anderson type models without a covering condition. Subsequently we showed in [3] how to use this approach to deduce localization for a great number of models that couldn't be treated before. Actually, for low energies we derived an uncertainty principle by an extremely simple method. However, for the link from these uncertainty principles to Wegner estimates with the correct volume factor we still had to rely upon the analysis of [5] which is technically quite involved. Here, we will use the fact that the uncertainty principles in [3] apply to the spectral projections of the random operators and not just to to the spectral projections of the underlying periodic background, as was the case in [5]. This allows the substantial shortcut we present in this paper.

To keep the paper selfcontained, we also present the necessary general spectral averaging result. Although this is basically wellknown, our discussion here seems to be somewhat more streamlined than what is available in the literature so far. Before stating the heart of the matter, we introduce and analyze the type of uncertainty principles that we referred to above.

This paper is dedicated to the late Pierre Duclos, whose untimely death we deeply mourn.

Acknowledgement. Fruitful discussions with Daniel Lenz and Ivan Veselić are gratefully acknowledged.

\section{An UNCERTAinty PRINCIPLE}

Here we just briefly review the uncertainty principles that came up in connection with random Schrödinger operators and mention relations to more classical uncertainty principles from Harmonic Analysis.

Roughly speaking, the classical uncertainty principle says that a function cannot be localized in position and momentum space at the same time. In what we consider here, momentum space does not necessarily refer to the usual Fourier transform.

Actually, we start in an abstract setting: $\mathcal{H}$ denotes a Hilbert space, $H$ a selfadjoint operator in $\mathcal{H}$, bounded from below and $0 \leq W$ a bounded operator on $\mathcal{H}$. By $I \subset \mathbb{R}$ we

Date: 21. 1. 2010. 
always denote an interval, and by $P_{I}(H)=\mathbb{1}_{I}(H)$ the corresponding spectral projection. The uncertainty principle we need is an estimate of the form

$$
P_{I}(H) W P_{I}(H) \geq \kappa P_{I}(H),
$$

with some $\kappa>0$. For the applications we have in mind, think of $H$ as a Schrödinger operator in $L^{2}$ and of $W$ as a function, that has to be spread out in a certain sense. If $W$ itself is bounded below by a strictly positive constant, $(\star)$ holds trivially. Let us record some easy facts in the following:

Proposition 2.1. $\quad$ (1) Denote the range of $P_{I}(H)$ by $\mathcal{H}_{I}$. Then $(\star)$ is equivalent to

$$
\left\|W^{\frac{1}{2}} f\right\|^{2} \geq \kappa\|f\|^{2} \text { for all } f \in \mathcal{H}_{I} .
$$

(2) If $(\star)$ holds and $J \subset I$ is a smaller interval, then $(\star)$ holds for $J$ in place of $I$.

(3) Abbreviate $P_{I}:=P_{I}(H), Q_{I}:=1-P_{I}$. If

$$
\left\|Q_{I} f\right\|^{2}+\left\|W^{\frac{1}{2}} f\right\|^{2} \geq \eta\|f\|^{2} \text { for all } f \in \mathcal{H}, \quad(\star \star)
$$

then $(\star)$ holds with $\kappa=\eta$. Conversely, if $(\star)$ holds for some $\kappa>0$, then $(\star \star)$ holds for some $\eta>0$.

In [3] we showed:

Theorem $2.2\left([3]\right.$, Theorem 1.1). Define $\lambda(t):=\inf \sigma(H+t W)$ for $t \geq 0$. If $\lambda\left(t_{0}\right)>E_{0}:=$ $\lambda(0)$ and $E_{1}<\lambda\left(t_{0}\right)$, then $(\star)$ holds for $I=\left[E_{0}, E_{1}\right]$ with

$$
\kappa=\frac{\lambda\left(t_{0}\right)-E_{1}}{t_{0}} .
$$

We now turn to the more specific situation that is relevant to the applications we have in mind: $\mathcal{H}=L^{2}\left(\mathbb{R}^{d}\right), H$ is a Schrödinger operator. Without too much restriction, we could even assume that $W=\mathbb{1}_{E}$, where $E \subset \mathbb{R}^{d}$ is a measurable set.

Let us first consider the case $H=-\Delta$. Then

$$
\mathcal{H}_{I}=\left\{f \in L^{2}\left(\mathbb{R}^{d}\right) \mid \operatorname{supp} \hat{f} \subset \Sigma_{I}\right\}, \text { where } \Sigma_{I}=\left\{\left.\xi \in \mathbb{R}^{d}|| \xi\right|^{2} \in I\right\}
$$

is an annulus or a ball. In virtue of Proposition 2.1 (1), the uncertainty principle $(\star)$ is equivalent to

$$
\|f\|_{L^{2}(E)}^{2} \geq \kappa\|f\|^{2} \text { if } \operatorname{supp} \hat{f} \subset \Sigma_{I} .
$$

In the language of uncertainty principles of Harmonic Analysis this latter inequality means that $E$ and $\Sigma_{I}$ form a strongly annihilating pair, cf [6]. Following [10] we say that $E$ is thick, provided there exist $a_{1}, \ldots, a_{d}>0$ and $\alpha>0$ such that for $C:=\left[0, a_{1}\right] \times \ldots \times\left[0, a_{d}\right]$ :

$$
|E \cap(C+y)| \geq \alpha \text { for all } y \in \mathbb{R}^{d},
$$

where $|\cdot|$ denotes the Lebesgue measure. The following generalization of the LogvinenkoSereda Theorem can be found in [10]:

Theorem 2.3 ([10], Theorem 4). For any bounded interval $I$ and any thick subset E:

$$
P_{I}(-\Delta) \mathbb{1}_{E} P_{I}(-\Delta) \geq \kappa P_{I}(-\Delta) .
$$


In fact, the statement in [10] is much more precise, the constant can be estimated from below and the $L^{2}$ estimate has an $L^{p}$ counterpart. Combined with the analysis from [5], the latter estimate can be used to prove continuity of the IDS and Wegner estimates at all energies for Anderson models with quite irregular geometry of the set of impurities. We do not pursue this issue here.

Evidently, the latter theorem was not known to Combes, Hislop and Klopp who proved a similar but somewhat different result with $H_{0}=-\Delta+V_{0}$, where $V_{0}$ and $W$ are periodic over the same lattice in [4]:

Theorem 2.4. Let $H_{0}=-\Delta+V_{0}$, with $V_{0}$ and $W$ periodic over the same lattice and $W \neq 0$ on some open set. Then

$$
P_{I}\left(H_{0}\right) W P_{I}\left(H_{0}\right) \geq \kappa P_{I}\left(H_{0}\right) .
$$

Let us now comment on how the above three uncertainty principles differ.

Remark 2.5. The above three theorems are mutually incomparable: as shown in [3], Theorem 2.2 applies in situations where $H=H(\omega)$ and $W$ may even be concentrated near a subspace of $\mathbb{R}^{d}$, so that $W$ is not "thick" at all; the decisive input is the mobility of the ground state energy. However, we can only treat intervals I close to the ground state energy.

In Theorem 2.3, $W$ maybe fairly general, $I$ is just supposed to be bounded, but $H=-\Delta$ is essential.

In Theorem 2.4. $H_{0}=-\Delta+V_{0}$ with a periodic $V_{0}, I$ is just supposed to be bounded, but $W$ has to be periodic.

\section{Spectral aVeraging for general measures.}

The main result here is, in fact, basically known although the formulation is sometimes slightly more complicated or restricted to a less general setting. We start with a real analysis lemma. Denote, for a probability measure $\mu$ on $\mathbb{R}$ the number

$$
s(\mu, \varepsilon):=\sup \{\mu([a, a+\varepsilon]) \mid a \in \mathbb{R}\},
$$

that is sometimes called the Lévy concentration function.

Lemma 3.1. For any probability measure $\mu$ on $\mathbb{R}, 0<\lambda \leq 1, a \in \mathbb{R}$ and $\varepsilon>0$ :

$$
\int_{\mathbb{R}} \frac{\lambda \varepsilon^{2}}{(x-a)^{2}+\lambda^{2} \varepsilon^{2}} d \mu(x) \leq 4 \frac{1}{\lambda} s(\mu, \varepsilon) .
$$

Proof. First observe that it suffices to treat $a=0$ and that

$$
\frac{\lambda \varepsilon^{2}}{x^{2}+\lambda^{2} \varepsilon^{2}} \leq \frac{1}{\lambda} \frac{\varepsilon^{2}}{x^{2}+\varepsilon^{2}} .
$$

Now we divide

$$
\mathbb{R}=\bigcup_{k \in \mathbb{Z}}\left[k \varepsilon-\frac{\varepsilon}{2}, k \varepsilon+\frac{\varepsilon}{2}\right]
$$


so that

$$
\begin{aligned}
\int_{\mathbb{R}} \frac{\varepsilon^{2}}{x^{2}+\varepsilon^{2}} d \mu(x) & \leq \sum_{k \in \mathbb{Z}} \int_{\left[k \varepsilon-\frac{\varepsilon}{2}, k \varepsilon+\frac{\varepsilon}{2}\right]} \frac{\varepsilon^{2}}{x^{2}+\varepsilon^{2}} d \mu(x) \\
& \leq s(\mu, \varepsilon)\left(1+2 \sum_{k \in \mathbb{N}} \frac{1}{\left(k-\frac{1}{2}\right)^{2}+1}\right),
\end{aligned}
$$

where we used the monotonicity of the integrand to estimate the integrals by the maximum of the integrand times the integral over $\left[k \varepsilon-\frac{\varepsilon}{2}, k \varepsilon+\frac{\varepsilon}{2}\right]$, which gives a factor of $s(\mu, \varepsilon)$. We can now compare the sum with the integral of $\left(x^{2}+1\right)^{-1}$ over $[1, \infty)$ plus an extra term to account for the first term in the sum. The best estimate we get in this fashion is $\left(\frac{9}{5}+\frac{\pi}{2}\right) s(\mu, \varepsilon)$ which proves the claim.

The following result can be seen as a streamlined version of Theorem 3.1 in [5]. We fix a Hilbert space $\mathcal{H}$ and denote its inner product by $(\cdot \mid \cdot)$.

Theorem 3.2. Let $\mu$ be probability measure on $\mathbb{R}, A$ a selfadjoint operator in $\mathcal{H}$ and $0 \leq B$ a bounded operator on $\mathcal{H}$. For any interval $I$ of length $|I|$ we get

$$
\int_{\mathbb{R}}\left(B^{\frac{1}{2}} P_{I}(A+t B) B^{\frac{1}{2}} \Phi \mid \Phi\right) d \mu(t) \leq 6\|B\|\|\Phi\|^{2} s(\mu,|I|),
$$

for any $\Phi \in \mathcal{H}$.

Proof. By functional calculus

$$
P_{I}(A+t B) \leq \frac{4}{\pi} \int_{I} d E\left\{\operatorname{Im}(A+t B-E-\mathrm{i} \varepsilon)^{-1}\right\},
$$

where we set $|I|=: \varepsilon$. Therefore,

$$
\left(B^{\frac{1}{2}} P_{I}(A+t B) B^{\frac{1}{2}} \Phi \mid \Phi\right) \leq \frac{4}{\pi} \int_{I} d E\left(B^{\frac{1}{2}} \operatorname{Im}(A+t B-E-\mathrm{i} \varepsilon)^{-1} B^{\frac{1}{2}} \Phi \mid \Phi\right) .
$$

As we can approximate $A, B$ we can assume without restriction that $A$ is bounded and $B$ is invertible. Thus we do not have any domain problems and we can write the integrand as

$$
\left(\operatorname{Im}\left[B^{-\frac{1}{2}}(A+t B-E-\mathrm{i} \varepsilon) B^{-\frac{1}{2}}\right]^{-1} \Phi \mid \Phi\right) .
$$

Next, we want to realize the inverse as the resolvent of some maximally accretive operator in order to be able to make use of the spectral theorem. (In [1] operators with strictly positive imaginary part are called dissipative; we are facing operators with strictly negative imaginary part so that accretive seems to be an appropriate term; note, however that these notions are not standardized at all.)

To do so, choose $\lambda=1$ if $B \leq \beta<1$ and $0<\lambda<\frac{1}{\|B\|}$ else. In any case, we get

$$
\left(\operatorname{Im}\left[B^{-\frac{1}{2}}(A+t B-E-\mathrm{i} \varepsilon) B^{-\frac{1}{2}}\right]^{-1} \Phi \mid \Phi\right)=\left(\operatorname{Im}\left[A_{E, \varepsilon, \lambda}+t-\mathrm{i} \lambda \varepsilon\right]^{-1} \Phi \mid \Phi\right),
$$

where

$$
A_{E, \varepsilon, \lambda}=B^{-\frac{1}{2}}(A-E-\mathrm{i} \varepsilon(1-\lambda B)) B^{-\frac{1}{2}}
$$

is maximally accretive. As discussed in [1], Appendix B and in [5], Section 3, the resolvent of a maximally accretive operator can always be written as the resolvent of a selfadjoint dilation. 
Therefore, we get an auxiliary Hilbert space $\mathcal{H}_{E, \varepsilon, \lambda}$ that contains $\mathcal{H}$ and a selfadjoint operator $L_{E, \varepsilon, \lambda}$ in $\mathcal{H}_{E, \varepsilon, \lambda}$ such that

$$
\left(\operatorname{Im}\left[A_{E, \varepsilon, \lambda}+t-\mathrm{i} \lambda \varepsilon\right]^{-1} \Phi \mid \Phi\right)=\left(\operatorname{Im}\left[L_{E, \varepsilon, \lambda}+t-\mathrm{i} \lambda \varepsilon\right]^{-1} \Phi \mid \Phi\right)
$$

which can be written in terms of the spectral measure $\rho_{E, \varepsilon, \lambda}^{\Phi}$ of $L_{E, \varepsilon, \lambda}$ in the state $\Phi$ as

$$
\ldots=\int_{\mathbb{R}} \frac{\lambda \varepsilon}{(x+t)^{2}+\lambda^{2} \varepsilon^{2}} d \rho_{E, \varepsilon, \lambda}^{\Phi}(x) .
$$

We are now almost ready to use the previous Lemma. Putting the above estimates together we get

$$
\int_{\mathbb{R}}\left(B^{\frac{1}{2}} P_{I}(A+t B) B^{\frac{1}{2}} \Phi \mid \Phi\right) d \mu(t) \leq \frac{4}{\pi} \int_{\mathbb{R}} \int_{I} d E \int_{\mathbb{R}} \frac{\lambda \varepsilon}{(x+t)^{2}+\lambda^{2} \varepsilon^{2}} d \rho_{E, \varepsilon, \lambda}^{\Phi}(x) d \mu(t)
$$

We can now use Fubini and the previous Lemma, giving

$$
\begin{aligned}
\ldots & \leq \frac{4}{\pi} \int_{I} d E \int_{\mathbb{R}} 4 \frac{1}{\lambda} s(\mu, \varepsilon) \frac{1}{\varepsilon} d \rho_{E, \varepsilon, \lambda}^{\Phi}(x) \\
& \leq 4 \frac{4}{\pi} \int_{I} d E \frac{1}{\lambda} s(\mu, \varepsilon) \frac{1}{\varepsilon}\|\Phi\|^{2}
\end{aligned}
$$

since the total mass of $\rho_{E, \varepsilon, \lambda}^{\Phi}$ is just $\|\Phi\|^{2}$. Now the length of $I$ cancels the $\frac{1}{\varepsilon}$ so that

$$
\cdots \leq 4 \frac{4}{\pi} \frac{1}{\lambda} s(\mu, \varepsilon)\|\Phi\|^{2} .
$$

Since $\frac{1}{\lambda}$ can be chosen arbitrarily close to $\|B\|$ we get the assertion.

As the reader can easily check, two special cases of the previous result stand out as particularly simple: for $B=1$ there is no need to pass to selfadjoint dilations; for $B$ a rank one operator, the Birman-Schwinger principle gives a scalar expression immediately so that one does not need to refer to spectral measures. See [11] for a discussion of the latter case and for uniformly $\alpha$-Hölder continuous measures.

The following reformulation of Theorem 3.2 is particularly neat. Recall that we write $\rho_{H}^{\Phi}$ for the spectral measure of the selfadjoint operator $H$ in the state $\Phi$.

Theorem 3.3. Let $\mu$ be probability measure on $\mathbb{R}, A$ a selfadjoint operator in $\mathcal{H}$ and $0 \leq B$ a bounded operator on $\mathcal{H}$. Then the measure

$$
\rho=\int_{\mathbb{R}} \rho_{A+t B}^{B^{\frac{1}{2}} \Phi} d \mu(t)
$$

satisfies

$$
s(\rho, \varepsilon) \leq 6\|B\|\|\Phi\|^{2} s(\mu, \varepsilon) .
$$

Note that the continuity of the averaged spectral measure does not depend on $A$ and its dependence on $B$ is very explicit and easy to control. The above mentioned special cases lead to: 
Corollary 3.4. (1) In the situation of the preceding theorem assume that, furthermore, $B=1$. Then

$$
s\left(\int_{\mathbb{R}} \rho_{A+t B}^{\Phi} d \mu(t), \varepsilon\right) \leq 6\|\Phi\|^{2} s(\mu, \varepsilon)
$$

(2) In the situation of the preceding theorem assume that, furthermore, $B=P_{\Phi}$ is the orthogonal projection onto the onedimensional space spanned by the normed $\Phi$. Then

$$
s\left(\int_{\mathbb{R}} \rho_{A+t B}^{\Phi} d \mu(t), \varepsilon\right) \leq 6 s(\mu, \varepsilon)
$$

\section{Wegner estimates}

Here we show that an uncertainty principle for random Hamiltonians implies Wegner estimates. We use some clever tricks borrowed from [5, 9] to show how to reduce the estimation of the trace of eigenprojectors to an application of Theorem 3.2 above. Note, however, that both the input, uncertainty principles for random operators, and the statement of the following result are new. To state our Wegner estimate we have chosen the following setup which can be regarded as a compromise between a general abstract theorem and an application to a specific type of random model. In fact, since the Wegner bound is dealing with a Hamiltonian on one particular cube, we fix an open cube in $\mathbb{R}^{d}$

$$
\Lambda=\Lambda_{L}(0)=\left(-\frac{L}{2}, \frac{L}{2}\right)^{d}
$$

with $L \in 2 \mathbb{N}+1$. Observe that

$$
\bar{\Lambda}=\bigcup_{k \in \Lambda \cap \mathbb{Z}^{d}} \overline{\Lambda_{1}(k)}
$$

Here is the

\section{Setup:}

(S1) $V_{0} \in L^{p}(\Lambda)$, where $p=2$ if $d \leq 3$ and $p>d$ if $d>3$ and $H_{0}:=-\Delta+V_{0}$ with Dirichlet boundary conditions.

(S2) $\mathcal{I}$ is a finite index set, $C_{U} \geq 0$ and

$$
U_{\alpha} \in L^{\infty}(\Lambda), \quad 0 \leq U_{\alpha} \leq C_{U}
$$

for all $\alpha \in \mathcal{I}$. Denote $\mathcal{I}_{k}:=\left\{\alpha \in \mathcal{I} \mid U_{\alpha} \chi_{\Lambda_{1}(k)} \neq 0\right\}$.

(S3) For the probability measure $\mathbb{P}$ on $\Omega=\prod_{\alpha \in \mathcal{I}}\left[0, \eta_{\text {max }}\right]$ we use regular conditional probabilities to define the following uniform bound

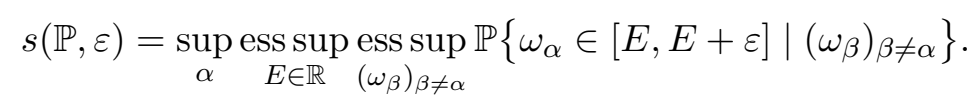

(See [8] for definition and existence of regular conditional probabilities.)

(S4) We set

$$
V_{\omega}:=\sum_{\alpha \in \mathcal{I}} \omega_{\alpha} U_{\alpha} \text { for } \omega=\left(\omega_{\alpha}\right)_{\alpha \in \mathcal{I}}
$$

and

$$
H:=H(\omega):=H_{0}+V_{\omega} .
$$


This setup typically arises when Anderson type random Schrödinger operators are restricted to cubes. Here is our main result:

Theorem 4.1. Let $H$ be as in the setup, $W:=\sum_{\alpha \in \mathcal{I}} U_{\alpha}$ and $I$ be some interval in $\mathbb{R}$. Assume that there is $\kappa>0$ such that $\mathbb{P}$-a.s.

$$
P_{I}(H) W P_{I}(H) \geq \kappa P_{I}(H) .
$$

Then, for a constant $C\left(V_{0}\right)$ that only depends on $V_{0}$,

$$
\mathbb{E}\left\{\operatorname{tr}\left[P_{I}(H)\right]\right\} \leq\left[C\left(V_{0}\right) \cdot C_{U}^{2} \cdot\left(\max _{k \in \Lambda \cap \mathbb{Z}^{d}}\left|\mathcal{I}_{k}\right|\right)\right] \kappa^{-2} \mathrm{e}^{\max I}|\Lambda| s(\mathbb{P},|I|) .
$$

Before proceeding to the proof let us comment on the estimate in the inequality (4.1) above. The term in square brackets is a structural constant that, typically, does not depend on $\Lambda$. As we showed in [3], we can find an uncertainty estimate of the form ( $\star$ ) with $\kappa$ independent of $\Lambda$ for very general Anderson type random models. The remaining terms give the right volume dependence as well as the continuity of the single site random variables.

We now single out several wellknown facts that will be used in the proof of the above theorem.

Remarks 4.2. (1) For operators on a general Hilbert space we record

(a) Cyclicity of the trace:

$$
\operatorname{tr}[A B]=\operatorname{tr}[B A],
$$

provided $A$ and $B$ are Hilbert Schmidt operators.

(b) For $A$ a selfadjoint Hilbert Schmidt operator, $0 \leq B \leq C$ bounded:

$$
\operatorname{tr}[A B A] \leq \operatorname{tr}[A C A] .
$$

(2) By monotonicity of the functional calculus,

$$
0 \leq P_{I}(H) \leq \mathrm{e}^{\max I} \mathrm{e}^{-H}
$$

for any semibounded selfadjoint $H$ and any bounded interval $I$ in $\mathbb{R}$.

(3) Denote

$$
H_{0}^{N}:=\bigoplus_{k \in \Lambda \cap \mathbb{Z}^{d}}\left(-\Delta+V_{0}\right)_{\Lambda_{1}(k)}^{N},
$$

the direct sum of $-\Delta+V_{0}$ restricted to the cube $\Lambda_{1}(k)$ with Neumann boundary conditions. Then

$$
H_{0}^{N} \leq H_{0} \leq H(\omega) \text { for all } \omega \in \Omega .
$$

(4) The operator $H_{0}^{N}$ has compact resolvent; denote, for $k \in \Lambda \cap \mathbb{Z}^{d}$, by $\Phi_{k, j}, j \in \mathbb{N}$, an orthonormal basis of eigenfunctions with corresponding eigenvalues $E_{k, j}$. Then

$$
\sum_{k, j} \mathrm{e}^{-E_{k, j}}=\operatorname{tr}\left[\mathrm{e}^{-H_{0}^{N}}\right] \leq C\left(V_{0}\right) \cdot|\Lambda| .
$$

For $V_{0} \equiv 0$ this can be read off explicit foemula for the eigenvalues, cf. [12], $p .266$. Under assumption (S1), $V_{0}$ is relatively bounded with respect to $-\Delta$ and so we get an estimate of the above type. 
Proof of Theorem 4.1. We start by using Remarks 4.2(1) and (2):

$$
\begin{aligned}
\operatorname{tr}\left[P_{I}(H)\right] & \leq \mathrm{e}^{\max I} \operatorname{tr}\left[P_{I}(H) \mathrm{e}^{-H} P_{I}(H)\right] \\
& =\mathrm{e}^{\max I} \operatorname{tr}\left[P_{I}(H) \mathrm{e}^{-H}\right]
\end{aligned}
$$

expanding into an orthonormal basis $\left(\varphi_{k}(\omega)\right)_{k \in \mathbb{N}}$ of $H(\omega)$ with eigenvalues $\mu_{k}(\omega)$ we obtain

$$
\begin{aligned}
\ldots & =\mathrm{e}^{\max I} \sum_{\mu_{k}(\omega) \in I} \mathrm{e}^{-\mu_{k}(\omega)} \\
& =\mathrm{e}^{\max I} \sum_{\mu_{k}(\omega) \in I} \mathrm{e}^{-\left(H(\omega) \varphi_{k}(\omega) \mid \varphi_{k}(\omega)\right)} \\
& \leq \mathrm{e}^{\max I} \sum_{\mu_{k}(\omega) \in I} \mathrm{e}^{-\left(H_{0}^{N} \varphi_{k}(\omega) \mid \varphi_{k}(\omega)\right)} \\
& \leq \mathrm{e}^{\max I} \sum_{\mu_{k}(\omega) \in I}\left(\mathrm{e}^{-H_{0}^{N}} \varphi_{k}(\omega) \mid \varphi_{k}(\omega)\right),
\end{aligned}
$$

where we used Remark 4.2(3) in the next to last and Jensen's inequality in the last step. This important trick is borrowed from [9]. We go on with

$$
\begin{aligned}
\ldots & =\mathrm{e}^{\max I} \operatorname{tr}\left[P_{I}(H) \mathrm{e}^{-H_{0}^{N}}\right] \\
& =\mathrm{e}^{\max I} \operatorname{tr}\left[\mathrm{e}^{-\frac{1}{2} H_{0}^{N}} P_{I}(H) \mathrm{e}^{-\frac{1}{2} H_{0}^{N}}\right] .
\end{aligned}
$$

We now estimate the latter expression, using the abbreviation $P_{I}:=P_{I}(H)$ and the main condition, $(\star)$ :

$$
\operatorname{tr}\left[\mathrm{e}^{-\frac{1}{2} H_{0}^{N}} P_{I} \mathrm{e}^{-\frac{1}{2} H_{0}^{N}}\right] \leq \kappa^{-1} \operatorname{tr}\left[\mathrm{e}^{-\frac{1}{2} H_{0}^{N}} P_{I} W P_{I} \mathrm{e}^{-\frac{1}{2} H_{0}^{N}}\right]
$$

Now comes a nice idea from [5]. We denote by $\|\cdot\|_{H S}$ the Hilbert-Schmidt norm and by $(\cdot \mid \cdot)_{H S}$ the corresponding inner product, given by $(A \mid B)_{H S}=\operatorname{tr}\left[B^{*} A\right]$ so that the Cauchy Schwarz inequality yields

$$
\begin{aligned}
\kappa^{-1} \operatorname{tr}\left[W P_{I} \mathrm{e}^{-H_{0}^{N}}\right] & =\kappa^{-1} \operatorname{tr}\left[\mathrm{e}^{-\frac{1}{2} H_{0}^{N}} P_{I} P_{I} W P_{I} \mathrm{e}^{-\frac{1}{2} H_{0}^{N}}\right] \\
& \leq \kappa^{-1}\left\|P_{I} W P_{I} \mathrm{e}^{-\frac{1}{2} H_{0}^{N}}\right\|_{H S}\left\|P_{I} \mathrm{e}^{-\frac{1}{2} H_{0}^{N}}\right\|_{H S} \\
& \leq \kappa^{-1}\left(\frac{c}{2}\left\|P_{I} W P_{I} \mathrm{e}^{-\frac{1}{2} H_{0}^{N}}\right\|_{H S}^{2}+\frac{1}{2 c}\left\|P_{I} \mathrm{e}^{-\frac{1}{2} H_{0}^{N}}\right\|_{H S}^{2}\right) \\
& =\kappa^{-1}\left(\frac{c}{2} \operatorname{tr}\left[P_{I} \mathrm{e}^{-H_{0}^{N}}\right]+\frac{1}{2 c} \operatorname{tr}\left[P_{I} W P_{I} W P_{I} \mathrm{e}^{-H_{0}^{N}}\right]\right)
\end{aligned}
$$


which holds for any $c>0$. We pick $c=\kappa$, insert (4.4) into (4.3) and move the first term in (4.4) to the left:

$$
\begin{aligned}
\operatorname{tr}\left[P_{I} \mathrm{e}^{-H_{0}^{N}}\right] & \leq \kappa^{-2} \operatorname{tr}\left[P_{I} W P_{I} W P_{I} \mathrm{e}^{-H_{0}^{N}}\right] \\
& =\kappa^{-2} \operatorname{tr}\left[P_{I} W P_{I} \mathrm{e}^{-H_{0}^{N}} P_{I} W P_{I}\right] \\
& \leq \kappa^{-2} \operatorname{tr}\left[P_{I} W \mathrm{e}^{-H_{0}^{N}} W P_{I}\right] \\
& \leq \kappa^{-2} \operatorname{tr}\left[\mathrm{e}^{-\frac{1}{2} H_{0}^{N}} W P_{I} W \mathrm{e}^{-\frac{1}{2} H_{0}^{N}}\right] .
\end{aligned}
$$

We now pick an eigenbasis as described in Remark 4.2(4) and get

$$
\begin{aligned}
\ldots & =\kappa^{-2} \sum_{k, j}\left(W P_{I} W \mathrm{e}^{-\frac{1}{2} H_{0}^{N}} \Phi_{k, j} \mid \mathrm{e}^{-\frac{1}{2} H_{0}^{N}} \Phi_{k, j}\right) \\
& =\kappa^{-2} \sum_{k, j} \mathrm{e}^{-E_{k, j}}\left(W P_{I} W \Phi_{k, j} \mid \Phi_{k, j}\right) \\
& =\kappa^{-2} \sum_{k} \sum_{j} \mathrm{e}^{-E_{k, j}} \sum_{\alpha, \alpha^{\prime} \in \mathcal{I}_{k}}\left(U_{\alpha} P_{I} U_{\alpha^{\prime}} \Phi_{k, j} \mid \Phi_{k, j}\right) .
\end{aligned}
$$

In the last step we used that $U_{\beta} \Phi_{k, j}=0$ for $\beta \notin \mathcal{I}_{k}$. Now we want to control the inner sum. Note that

$$
L^{\infty} \times L^{\infty} \rightarrow \mathbb{R},(U, V) \mapsto\left(U P_{I} V \Phi_{k, j} \mid \Phi_{k, j}\right)
$$

is a nonnegative bilinear form (on real-valued functions). The Cauchy-Schwarz inequality gives that

$$
\begin{aligned}
\left|\left(U P_{I} V \Phi_{k, j} \mid \Phi_{k, j}\right)\right| & \leq\left(U P_{I} U \Phi_{k, j} \mid \Phi_{k, j}\right)^{\frac{1}{2}} \cdot\left(V P_{I} V \Phi_{k, j} \mid \Phi_{k, j}\right)^{\frac{1}{2}} \\
& \leq \frac{1}{2}\left[\left(U P_{I} U \Phi_{k, j} \mid \Phi_{k, j}\right)+\left(V P_{I} V \Phi_{k, j} \mid \Phi_{k, j}\right)\right]
\end{aligned}
$$

so that

$$
\sum_{\alpha, \alpha^{\prime} \in \mathcal{I}_{k}}\left(U_{\alpha} P_{I} U_{\alpha^{\prime}} \Phi_{k, j} \mid \Phi_{k, j}\right) \leq 2 \sum_{\alpha \in \mathcal{I}_{k}}\left(U_{\alpha} P_{I} U_{\alpha} \Phi_{k, j} \mid \Phi_{k, j}\right) .
$$

Inserting into 4.6 and interchanging summation and expectation we get

$$
\begin{aligned}
\mathbb{E}\left\{\operatorname{tr}\left[P_{I}(H)\right]\right\} & \leq \mathrm{e}^{\max I} \mathbb{E}\left\{\operatorname{tr}\left[W P_{I} \mathrm{e}^{-H_{0}^{N}}\right]\right\} \\
& \leq 2 \mathrm{e}^{\max I} \kappa^{-2} \sum_{k} \sum_{j} \mathrm{e}^{-E_{k, j}} \sum_{\alpha \in \mathcal{I}_{k}} \mathbb{E}\left\{\left(U_{\alpha} P_{I} U_{\alpha} \Phi_{k, j} \mid \Phi_{k, j}\right)\right\},
\end{aligned}
$$

where we used the estimates 4.3 , 4.4 and 4.5 . Since $U_{\alpha} \leq C_{U} U_{\alpha}^{\frac{1}{2}}$ for all $\alpha$, we get

$$
\mathbb{E}\left\{\operatorname{tr}\left[P_{I}(H)\right]\right\} \leq 2 C_{U} \mathrm{e}^{\max I} \kappa^{-2} \sum_{k} \sum_{j} \mathrm{e}^{-E_{k, j}} \sum_{\alpha \in \mathcal{I}_{k}} \mathbb{E}\left\{\left(U_{\alpha}^{\frac{1}{2}} P_{I} U_{\alpha}^{\frac{1}{2}} \Phi_{k, j} \mid \Phi_{k, j}\right)\right\},
$$

which makes the expectation accessible to an application of Theorem 3.2. So consider,

$$
\mathbb{E}\left\{\left(U_{\alpha}^{\frac{1}{2}} P_{I} U_{\alpha}^{\frac{1}{2}} \Phi_{k, j} \mid \Phi_{k, j}\right)\right\}=\mathbb{E}\left\{\int_{0}^{\eta_{\max }}\left(U_{\alpha}^{\frac{1}{2}} P_{I}(H(\omega)) U_{\alpha}^{\frac{1}{2}} \Phi_{k, j} \mid \Phi_{k, j}\right) d \mathbb{P}\left(\omega_{\alpha} \mid\left(\omega_{\beta}\right)_{\beta \neq \alpha}\right)\right\} .
$$


The inner integral can be written as

$$
\int_{0}^{\eta_{\max }}\left(U_{\alpha}^{\frac{1}{2}} P_{I}\left(H_{0}+\sum_{\beta \neq \alpha} U_{\beta}+\omega_{\alpha} U_{\alpha}\right) U_{\alpha}^{\frac{1}{2}} \Phi_{k, j} \mid \Phi_{k, j}\right) d \mathbb{P}\left(\omega_{\alpha} \mid\left(\omega_{\beta}\right)_{\beta \neq \alpha}\right) .
$$

With $A=H_{0}+\sum_{\beta \neq \alpha} U_{\beta}, B=U_{\alpha}$, the substitution $t=\omega_{\alpha}$ and $\mu=\mathbb{P}\left(\cdot \mid\left(\omega_{\beta}\right)_{\beta \neq \alpha}\right)$ the latter integral is

$$
\int_{\mathbb{R}}\left(B^{\frac{1}{2}} P_{I}(A+t B) B^{\frac{1}{2}} \Phi_{k, j} \mid \Phi_{k, j}\right) d \mu(t) \leq 6 C_{U} s(\mu,|I|),
$$

since the $\Phi_{k, j}$ are normed. By definition, $s(\mu,|I|) \leq s(\mathbb{P},|I|)$, which gives an estimate independent of $k, j$. Inserting 4.8 into 4.8 we get

$$
\begin{aligned}
\mathbb{E}\left\{\operatorname{tr}\left[P_{I}(H)\right]\right\} & \leq 12 \cdot C_{U}^{2} \mathrm{e}^{\max I} \kappa^{-2} \sum_{k} \sum_{j} \mathrm{e}^{-E_{k, j}} \sum_{\alpha \in \mathcal{I}_{k}} s(\mathbb{P},|I|) \\
& \leq 12 \cdot C_{U}^{2} \mathrm{e}^{\max I} \kappa^{-2} \sum_{k} \sum_{j} \mathrm{e}^{-E_{k, j}}\left(\max _{k \in \Lambda \cap \mathbb{Z}^{d}}\left|\mathcal{I}_{k}\right|\right) s(\mathbb{P},|I|) \\
& \leq\left[C\left(V_{0}\right) \cdot C_{U}^{2} \cdot\left(\max _{k \in \Lambda \cap \mathbb{Z}^{d}}\left|\mathcal{I}_{k}\right|\right)\right] \kappa^{-2} \mathrm{e}^{\max I}|\Lambda| s(\mathbb{P},|I|),
\end{aligned}
$$

after using Remark 4.2(4), and incorporating 12 in the constant $C\left(V_{0}\right)$.

An immediate consequence is the usual form of Wegner estimates:

Corollary 4.3. Let $H$ be as in the setup, $W:=\sum_{\alpha \in \mathcal{I}} U_{\alpha}$ and $I$ be some interval in $\mathbb{R}$. Assume that there is $\kappa>0$ such that $\mathbb{P}$-a.s.

$$
P_{I}(H) W P_{I}(H) \geq \kappa P_{I}(H) .
$$

Then, for a structural constant $C$,

$$
\mathbb{P}\{\sigma(H(\omega)) \cap I \neq \emptyset\} \leq C \mathrm{e}^{\max I}|\Lambda| s(\mathbb{P},|I|) .
$$

For the proof, put the explicit constants into $C$ and note that

$$
\mathbb{1}_{\{\omega \mid \sigma(H(\omega)) \cap I \neq \emptyset\}} \leq \operatorname{tr}\left[P_{I}(H(\omega))\right],
$$

since $P_{I}(H(\omega))$ is a projection.

In 3 ] we showed that we have an uncertainty principle (太) with $\kappa$ independent of $\Lambda$. Moreover, for the models considered there, the other constants are uniformly bounded. Consequently, we get a fairly simple proof of Wegner estimates with a linear volume term. Note that Theorem 4.1 can also be used to obtain improvements (better volume factors) of some of the results in [7], notably of Theorem 8 and Corollary 9.

\section{REFERENCES}

[1] M. Aizenman, A. Elgart, S. Naboko, J. Schenker and G. Stolz. Moment Analysis for Localization in Random Schrödinger Operators. Invent. Math., 163: 343-413, 2006

[2] A. Boutet de Monvel, S. Naboko, P. Stollmann and G. Stolz. Localization near fluctuation boundaries via fractional moments and applications. J. Anal. Math., 100:83-116, 2006

[3] A. Boutet de Monvel, D. Lenz and P. Stollmann. An uncertainty principle, Wegner estimates and localization near fluctuation boundaries. Preprint arXiv:0905.2845 
[4] J.-M. Combes, P. D. Hislop, and F. Klopp. Hölder continuity of the integrated density of states for some random operators at all energies. Int. Math. Res. Not., 2003(4):179-209, 2003.

[5] J.-M. Combes, P. D. Hislop and F. Klopp. An optimal Wegner estimate and its application to the global continuity of the integrated density of states for random Schrödinger operators. Duke Math. J., 140(3):469498, 2007

[6] V.P. Havin and B. Jöricke. The Uncertainty Principle in Harmonic Analysis. Springer-Verlag, Berlin Heidelberg, 1994

[7] W. Kirsch and I. Veselić. Wegner estimates for sparse and other generalized alloy type potentials. Proc. Indian Acad. Sci. Math. Sci., 112(1):131-146, 2001

[8] A. Klenke. Probability theory. Universitext. Springer-Verlag London Ltd., London, 2008. A comprehensive course, Translated from the 2006 German original.

[9] F. Klopp and H. Zenk. The integrated density of states for an interacting multiparticle homogenous model and applications to the Anderson model. Advances in Mathematical Physics, Volume 2009, Article ID 679827, 15 pages, 2009. doi:10.1155/2009/679827

[10] O. Kovrijkine. Some results related to the Logvinenko-Sereda theorem. Proc. Am. Math. Soc., 129: 30373047,2001

[11] M. Krishna. Continuity of integrated density of states - independent randomness. Proc. Indian Acad. Sci. Math. Sci., 117(3):401-410, 2007

[12] M. Reed and B. Simon. Methods of Modern Mathematical Physics. IV: Analysis of Operators. Academic Press, New York, 1978

[13] I. Veselić. Existence and regularity properties of the integrated density of states of random Schrödinger operators. Volume 1917 of Lecture Notes in Mathematics. Springer-Verlag, Berlin, 2008

[14] F. Wegner. Bounds on the density of states in disordered systems. Z. Phys. B, 44(1-2):9-15, 1981

${ }^{\ddagger}$ Fakultät für Mathematik, Technische Universität, 09107 Chemnitz, Germany

E-mail address: peter.stollmann@mathematik.tu-chemnitz.de 